\title{
Planning Patient Care
}




\section{The Editors}

Lynn Batehup, BSc, MSc, RGN, is currently Clinical Lecturer in the Department of Nursing Studies, King's College, University of London, and at St George's Hospital.

Jenifer Wilson-Barnett, FRCN, PhD, RGN, is Professor of Nursing Studies at King's College, University of London.

\section{The Contributors}

Alison Jesson, RGN, HVCert, is currently Senior Nurse, Cardiac Counsellor and Specialist in Cardiac Rehabilitation at St George's Hospital, London.

Val Thomson, RGN, ADM, PGCEA, MTD, is currently a Midwife Tutor at the London Hospital.

Sue Adams, BSc, RGN, is currently a Sister in the Accident and Emergency Department at St George's Hospital, London.

Gary Tubman, RMN, is currently a Charge Nurse in the Community Psychiatric Department of St George's Hospital, London.

Sally Glen, RGN, RSCN, DN(Lond), RNT, DipEd(Lond), has been awarded a Health Education Council Scholarship and is currently studying at the Institute of Education, London.

Alison While, BSc, MSc, PhD, SRN, HVCert, is currently a Lecturer in the Department of Nursing Studies at King's College, University of London.

The illustrations on the front cover and on pages 1, 19, 45, 53, 67, 79 and 97 are by Duncan Smith. 


\title{
Planning Patient Care
}

\author{
Edited by
}

\section{Lynn Batehup}

Clinical Lecturer, Department of Nursing Studies

King's College, University of London

and

St George's Hospital, London

and

\section{Jenifer Wilson-Barnett}

Professor of Nursing Studies

King's College, University of London

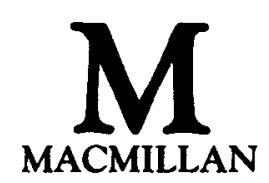


(C) Nursing Times, the Editors and the Contributors, 1988

All rights reserved. No reproduction, copy or transmission of this publication may be made without written permission.

No paragraph of this publication may be reproduced, copied or transmitted save with written permission or in accordance with the provisions of the Copyright Act 1956 (as amended), or under the terms of any licence permitting limited copying issued by the Copyright Licensing Agency, 33-4 Alfred Place, London WC1E 7DP.

Any person who does any unauthorised act in relation to this publication may be liable to criminal prosecution and civil claims for damages.

First published 1988

Published by

MACMILLAN EDUCATION LTD

Houndmills, Basingstoke, Hampshire RG21 2XS

and London

Companies and representatives

throughout the world

ISBN 978-0-333-46097-9

ISBN 978-1-349-10102-3 (eBook)

DOI 10.1007/978-1-349-10102-3 


\section{Contents}

The Editors and the Contributors ii

Introduction Lynn Batehup vii

1 Rehabilitation after a myocardial infarction:

Health education and counselling for long-

term recovery Alison Jesson

2 Helping a patient to understand diabetes:

Teaching plan to promote an independent

lifestyle Lynn Batehup

3 Assisting a mother to establish breastfeeding: Planning for a happy and successful postnatal period Val Thomson

4 A patient who has had cardiac surgery to relieve unstable angina: Planning in the acute recovery stage Lynn Batehup

5 An elderly lady who keeps falling: Planning for safe mobility Lynn Batehup

6 Symptom control for a patient with breast cancer: Planning for comfort Lynn Batehup

7 Drug overdose: Planning sensitive care in the accident and emergency department Sue Adams and Gary Tubman

8 Recovery from a stroke: Rehabilitation in the acute recovery stage Lynn Batehup

9 A patient who has had a lobectomy:

Planning in the acute post-operative period Sally Glen

10 A patient with heart failure: Planning care for a successful recovery Jenifer WilsonBarnett

11 A handicapped child admitted to hospital to improve her mobility skills: Planning care to improve walking and other self-care activities Alison While

12 Conclusion Lynn Batehup and Jenifer WilsonBarnett 


\section{Introduction}

$\mathrm{P}$ lanning patient care involves nurses in systematically assessing for and identifying a patient's problems, setting goals and outcomes, establishing interventions and evaluating the results. Planning is a universal concept that is used to ensure that future actions and results are those that are intended. The care plan is the tool used to document the process, to facilitate communication between care givers and to ensure continuity of care. The format that the care plan takes should be dictated by the needs of the individuals and the setting in which it is to be used. A certain rigidity exists regarding what data should be included in the care plan. There is no format that is more accept- able than any other. The main reason for the inclusion of any type of data in the care plan is that it should facilitate achievement of the patient's goals.

The format that is used for the plans in this book, is based on that proposed by Mayers (1983) and includes:

1. Assessment (gathering data, analysis and interpretation).

2. Identifying problems.

3. Defining expected outcomes (ongoing evaluation criteria).

4. Prescribing interventions (after considering options, constraints and resources).

5. Evaluation at periodic end point intervals.

\section{Assessment}

$\mathrm{P}$ lanned nursing care begins with an assessment. A thorough assessment provides data to enable the nurse to identify the problem areas. According to Christensen (1982), tools for data collection include interaction, observation and measurement. Making observations rather than drawing conclusions when collecting the data is important. Observations should be objective descriptions of behaviour; a conclusion is an interpretation, and it may therefore not be objective. Conclusions should not be drawn until all the available information is collected. Therefore, when carrying out an assessment, the nurse makes observations and uses communication to obtain information. More objectivity can be achieved if measurement is included, for example tracing and measuring an ulcer, or assessing pain with a pain ruler (Bourbonnais, 1981). By interpreting the information collected, the nurse can formulate perceptions about the patient and, where possible, get the patient to validate those perceptions. Theories, frameworks, models and principles are used as approaches to data collection (Christensen, 1982). These approaches give direction and guidance to the assessment process. Nursing models such as those described by Roy (1986), Orem (1985) and Roper et al. (1986) all provide parameters to aid patient assessment.

As a general rule, care plans in this book base the patient assessment on activities of daily living. The individual patient in most 
cases is the primary source of data. Other sources used to collect data include:

1. Family and friends.

2. Medical records.

3. Results of diagnostic tests.

4. Nursing records.

Assessment is an ongoing activity, part of the cycle of the nursing process. The assessment is followed by the nurse, or the nurse and the patient, arriving at one or more of the following conclusions:
1. No problem exists and the patient's health state is intact.

2. No problem exists but there is a potential problem.

3. There is a problem but the patient is coping effectively.

4. A problem exists that the patient needs help with handling.

5. A problem exists but needs further assessment.

\section{Problem statements}

T The problem is a concise statement of the patient's actual or potential health concern. Clarity and conciseness will help to ensure that the plan is communicated among the health carers. A specific and clearly stated problem is more likely to be read and understood than a lengthy paragraph that needs time to read and interpret. Terms that may be useful in stating patient problems include:

Inability to.

Lack of.

Unable to.

Difficulty in.

Potential for risk.

Impairment of.

Alteration in.

Failure to.

Limited.

Insufficient.

Inappropriate.

Reduced.

An example of a lengthy problem statement is 'Albert would like to stop smoking completely but feels that he needs cigarettes to calm him down. He has reduced from 30 to six a day'. This could be restated as 'Patient states failure to stop smoking following initial reduction from 30 to six a day'. The problem statement should also be specific and be patient centred. Complex problems should be broken down into smaller component parts for rational development of outcome criteria and interventions. For example 'Impairment of mobility related to thromboembolic brain damage' is too broad and general. This could be restated as 'Impaired voluntary movements of right limbs and trunk related to paralysis and spasticity' and 'Impaired postural balance control related to damage to nervous pathways'. If this is done, then specific and appropriate outcome criteria can be developed for each problem in contrast with vague and general goals or objectives, and long lists of interventions. It should be evident from the foregoing statements that the problem statement should be accompanied by the possible cause or causes and contributing factors. The aetiology of the problem will be directly related to the possible interventions that may be prescribed. For example 'Restricted joint movements related to inflamed joints' will necessitate interventions that may not be applicable to the problem 'Restricted joint movements related to hyperactivity of the spinal stretch reflex'. It may be helpful and improve clarity and continuity of care if the problem statement is accompanied by a brief statement of the subjective and objective data that identified it as a problem. For example the problem 'Impaired mobility related to painful swollen knees' would be accompanied by the findings:

1. Unable to extend knees fully.

2. Posture: knees bent, wide base, back bent.

3. States pain in knees is 5 on scale $0-10$. 
Many documentation systems have only an initial assessment document which is completed on or soon after admission. The continued identification of problems may be facilitated if a format that includes the subjective and objective assessment data for the problem could be recorded. As new problems are identified, they are accompanied by their assessment data. The problem list should reflect the patient's current health state. This entails regular assessment and update of the problem list.

\section{Outcomes}

Cas are planning requires that there be criteria against which the success or otherwise of the nursing interventions can be assessed or measured. Various terms have been used for this activity, including goals, aims and objectives. Unless it is explicitly stated, these terms do not actively facilitate the writing of the goal as the patient's goal but may allow the focus to be the nurses' goals. In this book the term 'expected outcome' (Mayers, 1983) is used throughout to refer to a statement of desired patient behaviours or clinical signs or symptoms that will indicate that the problem is being resolved or prevented. The expected outcome statement, like the problem statement, should be concise and specific and state clearly what patient behaviours or clinical signs and symptoms will represent alleviation of the problem if that is what is appropriate. The expected outcome statement will represent the criteria that are used to evaluate the effect of the nursing interventions. Mayers (1983) identifies four categories of possible patient outcomes:

1. Patients' self-report about what they know or understand, or how they feel about a specific situation or circumstance. This would include what the patient may say about his/her illness and treatment, or plans for the future. It would also include what the patient may say that he/she feels about for example coming into hospital, having an operation or being told his/her diagnosis. These expected outcome statements would usually start with 'Patient states: understanding of barium meal procedure'.
2. The patient's behaviour related to specific situations. This would refer to behaviours or activities that the nurse can observe, for example agitation and restlessness when first admitted to hospital, or an unsatisfactory technique for capillary blood glucose testing. The outcome statement may then start with the word 'Demonstrates', for example 'Demonstrates correct and accurate capillary blood glucose testing' or 'Demonstrates calm and relaxed posture'.

3. The patient's behaviours and symptoms related to the illness or disease process. This is reflected in laboratory and diagnostic tests, the patient's vital signs, and observing behaviours. These expected outcome statements usually reflect a desire to return to some stated 'norm' or baseline, for example 'Blood glucose values within the limits $3.5-5.0 \mathrm{mmol} /$ litre'. This may also include patient's self report, for example 'Patient states a reduction in pain felt from 6 to 0 on pain scale within 45 min' or 'Respiratory rate reduced from 40 to 20 / min within 2 hours'.

4. Expected outcome statements may be related to the patient's environment and would include all aspects which are of significance including family and significant others.

These four categories provide a general framework for possible ways of thinking how to write outcome statements that are patient oriented. However, there is considerable overlap between categories, and it may be less confusing to think of patient outcomes as what patients do and say and how they feel, and clinical manifestations that reflect 
the patient's health status. There may be combinations of these types of outcome, for example the problem 'Knowledge deficit related to diabetic self-management'. The expected outcomes will be a combination of what the patient says (knowledge about insulin overdose), what the patient demonstrates (correct examination of the feet) and physiological parameters (blood glucose values). Each expected outcome statement should include checking intervals for evaluation. There is little research evidence at present that will help nurses to make predictions about the rate that outcomes for various problems will be achieved. However, it is important that nurses start to make these predictions, and to record in the evaluation the outcome. This is then a systematic record that may be used in future research to identify realistic outcomes and successful interventions. Where possible, the expected outcome should always be discussed and agreed with the patient and family. Research by Horsley (1982) has shown that collaboration with patients can lead to more effective attainment of goals and improved patient satisfaction.

\section{Interventions}

T约

The nursing interventions are selected to help to alleviate the identified problem, and to move the patient towards meeting the stated expected outcomes. The intention is usually to direct the intervention towards altering or alleviating the cause or causes of the problem. This is not always possible, and it is then appropriate to direct the intervention to changing or treating the results or signs and symptoms of the problem. For example 'Impaired mobility related to damage to nervous pathways' will not enable interventions to be directed towards the cause (the damage to nervous pathways), and in this case the interventions will be related to the manifestations of that damage-paralysed limbs and increasing spasticity. However, the problem 'Impaired mobility related to painful knee joints' does enable the intervention to be aimed at the cause of the problem- reduction or removal of the pain.

In this book the sections entitled 'Rationale' provide the research base for the interventions where this is available. Many interventions prescribed and carried out by nurses and other health care providers do not have an apparent research base. Some are 'triedand-tested' remedies which seem to work, and some may be based on nothing more than 'folklore'. Some nurses have started to try to identify valid interventions for frequently occurring patient problems (Haller $e t$ al., 1979; Bulechek and McCloskey, 1985), and doubtless this will continue to take place in the future. Interventions may not always represent independent nursing activities but may be in collaboration with, or dependent upon, other health care providers such as therapists and doctors.

\section{Evaluation}

T The expected outcomes which are stated following the assessment and problem identification act as the criteria against which to judge the success of the nursing interventions. The evaluation may be written as the patient's actual outcome in relation to the interventions prescribed. The outcome statement should be written using the criteria in the expected outcome statement as a guide. For example, if the expected outcome is 'Pressure sore to decrease in size from $45 \mathrm{~mm}$ to $40 \mathrm{~mm}$ ' and this is reviewed at 
5-day intervals, then the evaluation or actual outcome statement should state the new measurement and whether or not this is a reduction in size. If the expected outcome was written so as to reflect the patient's statement or behaviour, or the environmental situation, then the actual outcome statement can be written in the same way. The actual or observed patient responses to care are factual statements which, when compared with the expected outcome statement, give a fairly clear indication of the success or failure of the prescribed nursing interventions. Evaluation may have several outcomes:

1. The expected outcomes have been achieved by the patient and the problem has been resolved.

2. The expected outcomes have been achieved by the patient but the problem needs to be monitored further.

3. The expected outcomes have not been realised and so the situation needs to be reassessed:

a. Was the problem identified the correct one?

b. Is the cause of the problem still the same?

c. Are the contributing factors still the same?

d. Was the problem properly assessed in the first place?

e. Were the best or correct interventions selected?

f. Were the interventions consistently implemented?

g. Were the expected outcomes realistic or appropriate?

h. Has there been sufficient time to achieve the outcomes?

As was stated earlier, careful recording of actual outcomes in relation to prescribed interventions will provide a rich source of data for researchers to analyse and identify effective and ineffective interventions which may then be subjected to further evaluation.

The nursing process or problem-solving approach, it is argued by Henderson (1982), is not the only approach in nursing. Henderson (1982) is critical of the view that logical deduction is the only way to solve problems and puts a case for the validity of intuitive judgement derived from clinical knowledge as a mode of problem solving which is of equal value to nurses. There is evidence that the more experienced expert nurse is able to grasp the intricacies of a clinical situation rapidly and can sort out relevant from irrelevant information with a well-developed perceptual awareness (Benner, 1984). These nurses are distinguished from the beginner who must rely on a deliberate analytical method to build a clinical picture from isolated pieces of information. Therefore, it becomes important that a systematic framework such as the nursing process is used by nurses and that it is recognised that the nurse's experience and ability will determine how it is used. There is evidence that nurses may be collecting assessment data but for several reasons are failing to examine relationships within the data and so often make poor judgements or do not arrive at appropriate conclusions (Field, 1983). There may therefore be a special need for nurses to be taught how to analyse and interpret assessment data. Therefore, throughout this book, each care plan involves the reader in some interpretation of the assessment data to identify further problems not included in the main care plan. In addition, the reader is also asked to think about other interventions and evaluation criteria which may be relevant to problems already identified. All the care plans relate to 'real' patients. Answers to reader questions are printed on a separate page. All possible problems are not included in the care plans; so it is hoped that the reader will go on to expand the plans and that they are used as teaching tools in wards and classrooms. 


\section{References}

Benner, P. (1984). From Novice to Expert, Addison-Wesley, Menlo Park, California.

Bourbonnais, F. (1981). Pain assessment: development of a tool for the nurse and patient. Journal of Advanced Nursing, 6, 277282.

Bulechek, G. M., and McCloskey, J. C. (1985). Nursing Interventions. Treatments for Nursing Diagnoses. W. B. Saunders, Philadelphia, Pennsylvania.

Christensen, P. (1982). Nursing Assessment: data collection of the individual patient. In: Griffith, J. W., and Christensen, P. J. (eds), Nursing Process. Application of Theories, frameworks, and models, C. V. Mosby, St Louis, Missouri.

Field, P. A. (1983). An ethnography: four public health nurses' perceptions of nursing. Journal of Advanced Nursing, 8, 3-12.

Haller, K. B., Reynolds, M. A., and Horsley, J. A. (1979). De- veloping research based innovation protocols: process criteria, and issues. Research in Nursing and Health, 2, 45-51.

Henderson, V. (1982). The nursing process-is the title right? Journal of Advanced Nursing, 7, 103-116.

Horsley, J. A. (1982). Mutual goal setting in patient care. Conduct and Utilisation of Research in Nursing Project, Michigan Nurses Association, Grune and Stratton, New York.

Mayers, M. (1983). A Systematic Approach to the Nursing Care Plan, Appleton Century Crofts, Norwalk, Connecticut.

Orem, D. (1985). Nursing: Concepts of Practice, McGraw-Hill, New York.

Roper, N., Logan, W. W., and Tierney, A. J. (1986). The Elements of Nursing, Churchill Livingstone, Edinburgh.

Roy, C. (1986). Introduction to Nursing, an adaptation model, PrenticeHall, Englewood Cliffs, New Jersey. 